\title{
EPOC, BRONQUITIS CRÓNICA Y SÍNTOMAS RESPIRATORIOS, ASOCIADOS A LA CONTAMINACIÓN POR PM10 EN LA CIUDAD DE MEDELLÍN (COLOMBIA)
}

\author{
Carlos Eduardo Orduz García, MD, MPH, FCCP \\ María Victoria TORO \\ Juan Carlos Gómez, \\ ${ }^{1}$ Internista neumólogo, profesor titular de medicina, Universidad Pontificia Bolivariana. \\ ${ }^{2}$ Ingeniera ambiental, profesora asociada UPB PhD ingeniería ambiental. \\ ${ }^{3}$ Ingeniero ambiental, MPH ingeniería Ambiental GIA, Grupo de Investigación Ambiental, CIDI, UPB, Grupo B de Colciencias.
}

Recibido: Noviembre 15 de 2012 Aceptado: Enero 21 de 2013

\section{Resumen}

Introducción: Existe una evidencia creciente de que la contaminación aérea en las ciudades tiene efectos de larga duración sobre la enfermedad respiratoria crónica en niños, pero hay pocos estudios con resultados no concluyentes en adultos. Nosotros examinamos las asociaciones entre la contaminación por PM10 y los síntomas respiratorios, la bronquitis crónica y el EPOC en la ciudad de Medellín (Colombia). Para saber si estos niveles se asocian a mayor prevalencia de enfermedades respiratorias crónicas en adultos. Objetivos: Evaluar la prevalencia de síntomas respiratorios, bronquitis crónica y EPOC en estas dos áreas. Comparar las prevalencias de estas patologías en las dos áreas. Comparar los riesgos relativos y determinar si su diferencia es estadísticamente representativa. Diseño: Se diseñó un estudio de corte transversal con un componente analítico. Métodos: La investigación se hizo en dos fases. En la primera (fase I) se evaluaron los aspectos ambientales y se seleccionaron las áreas de alta y baja contaminación de la ciudad por PM10. En la segunda (fase II) se diseñó una investigación epidemiológica de corte transversal con un componente analítico, se usó un cuestionario y se le realizo una espirometria antes y después de aplicar 200 ug de salbutamol inhalado, según el protocolo de la ATS/ERS. Las definiciones operativas que usamos son las siguientes: bronquitis crónica: tos y flema diariamente por tres meses durante dos años consecutivos. EPOC: VEF1/CVF $<70 \%$ postbroncodilatador. Síntomas respiratorios: tos, flema, sibilancias, disnea. El análisis se realizó usando un software SPSS. Resultados: Se incluyeron un total de 578 sujetos, 381 en el área de Guayabal y 197 sujetos en el área de Robledo. Después del análisis estadístico, se encontró que vivir por más de cinco (5) años en área con niveles de contaminación por PM10 de $75 \mathrm{ug} / \mathrm{m} 3$ en promedio, comparado con vivir por más de cinco (5) años en área con niveles de $45 \mathrm{ug} / \mathrm{m} 3$, está asociado con una mayor prevalencia de síntomas respiratorios de tos y flema, bronquitis crónica, obstrucción bronquial y EPOC. Conclusiones: Vivir en área contaminada por PM10, durante 5 años o más, está asociada a una mayor prevalencia de síntomas respiratorios, bronquitis crónica y EPOC. El riesgo relativo de desarrollar EPOC es significativamente mayor en el área de niveles altos de contaminación por PM10. Ésto indica que la contaminación aérea por PM10 tiene efectos de larga duración sobre la enfermedad respiratoria crónica en adultos. Estudios adicionales se requieren para confirmar estos hallazgos.

Palabras clave: Bronquitis crónica, EPOC, síntomas respiratorios, epidemiologia, contaminación ambiental aérea. 
EPOC, BRONQUITIS CRÓNICA Y SÍNTOMAS RESPIRATORIOS, ASOCIADOS

a la CONTAMinación por PMio en la CiUdad de Medellín (Colombia)

\title{
COPD, CHRONIC BRONCHITIS AND RESPIRATORY SYMPTOMS ASSOCIATED WITH PM10 POLLUTION IN THE CITY OF MEDELLIN (COLOMBIA)
}

\begin{abstract}
There is increasing evidence that air pollution in cities has long-term effects on chronic respiratory disease in children, yet there are scarce studies with non conclusive results in adults. We examined the associations between pollution resulting from PM10 and respiratory symptoms, chronic bronchitis and COPD in the city of Medellín (Colombia) in order to determine if these levels were associated with a higher prevalence of adult chronic respiratory diseases. The objectives of this study were: to assess the prevalence of respiratory symptoms, chronic bronchitis and COPD in these two areas, to compare the prevalence of these pathologies between the two areas, and make a comparison of the relative risks in order to determine if such difference is statistically significant. A cross-sectional study with an analytical component was designed. The research was performed in two stages. In the first stage (Phase I) the environmental issues were assessed and the areas of high and low pollution caused by PM10 were selected. In the second stage (Phase II) a cross-sectional epidemiological investigation with an analytical component was designed. A questionnaire was used and a spirometry was performed before and after administering inhaled salbutamol $200 \mathrm{mg}$, accordingly to ATS/ERS protocol. The used operative definitions were as follows: Chronic bronchitis: cough and phlegm for three months during two consecutive years. COPD: FEV/FVC $<70 \%$ post-bronchodilator. Respiratory symptoms: Cough phlegm, wheezing, dyspnea. The analysis was performed using a SPSS software. A total of 578 subjects (381 in the area of Guayabal and 197 subjects in the area of Robledo) were included. Following the statistical analysis, it was found that living for over five (5) years in an area with average pollution by PM10 levels of $75 \mathrm{mg} / \mathrm{m}^{3}$, in comparison with living for more than five (5) years in an area with levels of $45 \mathrm{mg} / \mathrm{m}^{3}$ is associated with a higher prevalence of respiratory symptoms namely cough and phlegm, chronic bronchitis, bronchial obstruction and COPD. It was concluded that living in an area polluted with PM10 during 5 years or more is associated with higher prevalence of respiratory symptoms, chronic bronchitis and COPD. The relative risk of developing COPD is significantly higher in the area with higher levels of pollution by PM10. This shows that air pollution resulting from PM10 has long-term effects on adult chronic respiratory disease. Additional studies are required to confirm these findings.
\end{abstract}

Key words: Chronic bronchitis, COPD, Respiratory symptoms, Epidemiology, Environmental Air Pollution.

\section{EPOC, BRONQUITE CRÔNICA E SINTOMAS RESPIRATÓRIOS, ASSOCIADOS À CONTAMINAÇÃO POR PM 10 NA CIDADE DE MEDELLÍN (COLÔMBIA)}

\begin{abstract}
Resumo
Existe uma evidência crescente de que a contaminação aérea nas cidades tem efeitos de longa duração sobre a doença respiratória crônica em crianças, mas há poucos estudos com resultados não concluintes em adultos. Nós examinamos as associações entre a contaminação por PM 10 e os sintomas respiratórios, a Bronquite crônica e o EPOC na cidade de Medellín (Colômbia). Para saber se estes níveis se associam a maior prevalência de Doenças Respiratórias crônicas em adultos. Os objetivos do apresente estudo são avaliar a prevalência de Sintomas Respiratórios, Bronquite Crônica e EPOC nestas duas áreas, comparar as prevalências destas patologias nas duas áreas e comparar os riscos relativos
\end{abstract}


para determinar se a sua diferença é estatisticamente representativa. Desenhou-se um estudo de corte transversal com um componente analítico. A pesquisa se fez em duas fases. Na primeira (Fase I) se avaliaram os aspectos ambientais e se selecionaram as áreas de alta e baixa contaminação da cidade por PM10. Na segunda (Fase II) se desenhou uma pesquisa epidemiológica de corte transversal com um componente analítico. Usou-se um questionário e se lhe realizou uma espirometria antes e depois de aplicar $200 \mu \mathrm{g}$ de salbutamol inalado, segundo o protocolo da ATS/ERS. As definições operativas que usamos são as seguintes: Bronquite crônica: tosse e fleg diariamente por três meses durante dois anos consecutivos. EPOC: VEF1/CVF < 70\% pós broncodilatador. Sintomas respiratórios: tosse, flegma, sibilâncias, dispneia. A análise se realizou usando um software SPSS. Se incluiram um total de 578 sujeitos (381 na área de Guayabal e 197 sujeitos na área de Robledo. Depois da análise estatística se encontrou que morar por mais de cinco (5) anos em área com níveis de contaminação por PM 10 de $75 \mu \mathrm{g} / \mathrm{m} 3$ em média, comparado com morar por mais de cinco (5) anos em área com níveis de $45 \mu \mathrm{g} /$ $\mathrm{m} 3$ está associado com uma maior prevalência de sintomas respiratórios de tosse e flegma, bronquite crônica, obstrução bronquial e EPOC. A conclusão à que se chegou foi que morar em uma área contaminada por PM 10 durante 5 anos ou mais, está associada a uma maior prevalência de Sintomas respiratórios, Bronquite Crônica e EPOC. O risco relativo de desenvolver EPOCO é significativamente maior na área de níveis altos de contaminação por PM10. Isto indica que a contaminação aérea por PM 10 tem efeitos de longa duração sobre a doença respiratória crônica em adultos. Estudos adicionais se requerem para confirmar estas descobertas.

Palavras chave: Bronquite crônica, EPOC, sintomas respiratórios, Epidemiologia, Contaminação ambiental aérea.

\section{Introducción}

La contaminación aérea en las ciudades se ha asociado a efectos de corta duración y enfermedad respiratoria crónica, exacerbación y consultas a urgencias y hospitalizaciones por causas respiratorias $(1,2)$. También, con el aumento de los síntomas con niveles bajos de contaminación(3). Los efectos de larga duración son controvertidos pero hay una creciente evidencia de que la contaminación, al menos en los niños se asocia a la incidencia de asma $(4,5)$, desarrollo de una disminución de la función pulmonar $(6,7)$ e incidencia de síntomas de Bronquitis $(8,9)$.

En los adultos, los estudios de los efectos de larga duración han sido pocos, y estudios recientes han encontrado hallazgos tanto positivos $(10,11)$ como negativos $(12,13)$ asociados con asma y positivos $(14$, $15,16)$ y negativos $(17,18)$ asociados con epoc. Por otra parte, la enfermedad respiratoria crónica en adultos es heterogénea e incluye otras exposiciones, tales como el cigarrillo y exposición ocupacional, las cuales no afectan directamente a los niños. Esta gran variedad de factores de riesgo puede complicar la investigación y contribuir a resultados no concluyentes en adultos.
En este estudio, nosotros usamos la información de los niveles de contaminación por PM10 extraída de la evaluación realizada por nuestro grupo de ingeniería ambiental (GIA-UPB), (fase I de esta investigación), en un área de "alta" contaminación (Comuna de Guayabal) donde se encontró un promedio de PM10 de 75 ug/ $\mathrm{m} 3$, el cual estaba por encima del límite permisible (50 ug/m3) y otra área de "baja" contaminación (Comuna de Robledo) donde se encontró un promedio de PM 10 de $45 \mathrm{ug} / \mathrm{m} 3$, contaminación por debajo de los límites permisibles (19).

El objetivo de este estudio (fase II) fue investigar la asociación entre los niveles de contaminación por PM10 en dos áreas de la ciudad de Medellín (alta y baja contaminación) y la prevalencia de síntomas respiratorios, bronquitis crónica y EPOC en adultos y el riesgo relativo de vivir en estas dos aéreas para desarrollar estas enfermedades, y su asociación con las concentraciones de PM10, utilizando como criterio diagnostico del EPOC, la espirometria.

\section{Diseño}

Se diseñó un estudio de corte transversal con un componente analítico de dos fases. 
EPOC, BRONQUITIS CRÓNICA Y SÍNTOMAS RESPIRATORIOS, ASOCIADOS

a la CONTAMinación por PMio en la CiUdad de Medellín (Colombia)

\section{Metodología}

En la fase I se evaluó la calidad del aire en las comunas de Guayabal y de Robledo, las cuales en estudios de monitoreo de la calidad de aire de RED AIRE que se realiza en la ciudad de Medellín desde hace 10 años, habían mostrado tener niveles altos y bajos de contaminación aérea (20).

Estudio de población: Se seleccionó una muestra aleatoria simple, representativa de la población mayor de 40 años, con un nivel de confianza del 95\%, de 381 individuos en el área de Guayabal, y 197 en el área de Robledo que estuvieran viviendo o trabajando mínimo 8 horas en el día en estas áreas durante 5 años o más y que no tuvieran antecedentes de tabaquismo activo o pasivo, exposición ocupacional en oficios asociados con estas patologías, que pudieran responder al cuestionario y pudieran realizar la espirometría.

Criterios de inclusión: personas mayores de 40 años, residentes o trabajadoras por cinco años o más, que hayan resultado seleccionadas aleatoriamente de acuerdo con el diseño estadístico.

Criterios de exclusión: tener menos de 5 años de residencia o trabajo en el sitio. Personas fumadoras, ex fumadoras o fumadores pasivos. Personas que hayan laborado en canteras, textileras, cementeras, o expuestos laboralmente a químicos, polvos, humos, gases, vapores. Personas que hayan sido diagnosticadas con algún tipo de enfermedad respiratoria. Discapacidad física o mental que le impida a la persona responder las encuestas y participar en la realización de las espirometrías.

Se aplicó el cuestionario ATS-DLD-78 de Ferris (21) previamente validado y después de una prueba piloto, a la muestra seleccionada de cada área de estudio. La encuesta fue aplicada por 3 encuestadores previamente entrenados y se realizó un control de calidad a 1 de cada 20 entrevistados. Se realizaron las espirometrías con un equipo estándar y utilizando los criterios de calidad ATS/ERS para el equipo y la prueba. A quienes en la espirometría tamiz se les encontró obstrucción, se les realizó espirometría pos broncodilatador utilizando $200 \mathrm{mg}$ de salbutamol (ventilan) previo consentimiento informado (22). Los datos del cuestionario y de la espirometría fueron vaciados en una base de datos diseñada para esta investigación y posteriormente se realizó el análisis estadístico utilizando un paquete SPSS.
La información del estudio de población fue recolectada entre los meses de agosto a diciembre de 2007. Para el análisis de los datos se usaron las siguientes definiciones operativas: EPOC por espirometría: VEF1/ CVF $<70 \%$ pos broncodilatador. Gravedad del EPOC: se utilizaron las escalas Gold I para vef $1 / \mathrm{cvf}>80 \%$, II vef $1 /$ cvf $<$ de $80 \%$ y $>$ de $50 \%$, III vef $1 /$ cvf $<50 \%>$ $30 \%$, IV vef $1 /$ cvf $<$ de $30 \%$. Obstrucción Bronquial: vef $1 /$ cvf $<$ de $70 \%$ pre broncodilatador

Síntomas: Respuesta si a las preguntas: ¿Ha tenido: a) Tos frecuente, b) flema frecuente, c) alguna vez sibilancias (silbido, chillido, hervidero) en el pecho?; d) Disnea si respondió si a la pregunta: ¿se ahoga (se asfixia o le falta el aire) con cualquier actividad o en reposo?

Bronquitis crónica: Tos y expectoración $\geq 3$ meses por 2 años consecutivos.

Se utilizó una prueba de $\mathrm{CHI}$ cuadrado y test exacto de Fisher. Se determinó el RR para los síntomas y las patologías determinadas con las definiciones operativas en cada área y se estableció la diferencia con sus respectivas significancias estadísticas.

\section{Resultados de la fase II estudio en las personas}

El resultado de las características de la población estudiada en cada zona, la cual fue seleccionada aleatoriamente tiene un alto grado de homogeneidad, por rangos de edad, en cuanto a la ocupación, al grado de escolaridad, el tiempo de residencia o trabajo en la zona y el estrato socioeconómico. Se encontró que la población de 40 a 50 años en guayabal fue del $29 \%$ y en Robledo del 30\%, la población entre los 50 a 60 años fue del $28 \%$ en Guayabal y del $25 \%$ en Robledo, la población entre los 60 a 70 años fue de $25 \%$ en Guayabal y del $25 \%$ en Robledo y la población mayor de 70 años fue de $18 \%$ en Guayabal y en Robledo fue del $21 \%$.

Características de la ocupación en Guayabal: la distribución fue de un $48.5 \%$ amas de casa, el $17.8 \%$ son empleados, el $17.3 \%$ trabajadores independientes, el $12.9 \%$ pensionado/jubilado y el $3.7 \%$ otros, y en Robledo: la distribución fue de un $52.8 \%$ amas de casa, $12.7 \%$ empleados, $19.3 \%$ trabajadores independientes, $11.2 \%$ pensionado/jubilado y el $4.1 \%$ otros. Se encontró que el grado de escolaridad entre Guayabal y Robledo fue: ninguno en un $3 \%$ en Guayabal y un $5 \%$ en Robledo, primaria $45 \%$ Guayabal y $59 \%$ 
Robledo, secundaria $15 \%$ Guayabal y $24 \%$ Robledo, Técnico-tecnología $24 \%$ guayabal y $24 \%$ Robledo, Universitario 7\% Guayabal y 3\% Robledo y otros, $5 \%$ Guayabal y 0\% Robledo. El tiempo de residencia de 5 a 15 años fue de $25.2 \%$ en Guayabal y $38.6 \%$ en Robledo, de 15 a 30 años es de $21.5 \%$ en Guayabal a $32.5 \%$ Robledo, mayor de 30 años es de $53.3 \%$ en Guayabal y $28.9 \%$ en Robledo (tabla 1).

Resultados espirometricos: La prevalencia de alteración espirométrica obstructiva en Guayabal fue del $56(14,7 \%)$ y en Robledo del $14(7 \%)$ con un riesgo relativo de enfermar de $2,252 \mathrm{p}<0,001,95 \%(1,220$ 4,158 ) mayor en Guayabal que en Robledo (tabla 2).
La prevalencia de EPOC en Guayabal fue del 32 (8,4\%) y en Robledo fue de 7 (3,6\%), con un riesgo relativo (RR) de enfermar de 2,489 mayor en Guayabal que en Robledo de 2,489 95\% p < 0,024 (1,078-5,746) (tabla 2). No solo la prevalencia es mayor en Guayabal que en Robledo sino que además los casos son más graves en Guayabal que en Robledo. La prevalencia de bronquitis crónica (tos y flema por 3 meses durante 2 años) en Guayabal fue del 38 (9,97\%) y en Robledo de $8(4,06 \%)$ también mayor en Guayabal. Las exacerbaciones fueron más frecuentes en Guayabal 31 $(8,1 \%)$ frente a Robledo $5(2,53 \%)$ (tabla 3$)$. En las tablas se resumen estos resultados.

Tabla 1. Características sociodemográficas de la población estudiada

\begin{tabular}{|c|c|c|c|c|c|c|}
\hline \multicolumn{5}{|c|}{ EDAD } & & \\
\hline & 40 a 50 años & 50 a 60 años & 60 a 70 años & $<$ de 70 años & & \\
\hline GUAYABAL & $112(29 \%)$ & $107(28 \%)$ & $99(25 \%)$ & $63(18 \%)$ & & \\
\hline ROBLEDO & $61(30 \%)$ & $49(25 \% 9)$ & $46(25 \%)$ & $41(21 \%)$ & & \\
\hline \multicolumn{6}{|c|}{ OCUPACIÓN } & \\
\hline & Amas de casa & Empleados & Independientes & Pensionado & Otros & \\
\hline GUAYABAL & $48,50 \%$ & $17,80 \%$ & $17,30 \%$ & $12,90 \%$ & $3,70 \%$ & \\
\hline ROBLEDO & $52,80 \%$ & $12,70 \%$ & $19,30 \%$ & $11,20 \%$ & $4,10 \%$ & \\
\hline \multicolumn{7}{|c|}{ GRADO DE ESCOLARIDAD } \\
\hline & Ninguno & Primaria & Secundaria & Tecnico- tecno & Universitario & Otr \\
\hline GUAYABAL & $3 \%$ & $45 \%$ & $15 \%$ & $24 \%$ & $7 \%$ & $5 \%$ \\
\hline ROBLEDO & $5 \%$ & $59 \%$ & $24 \%$ & $24 \%$ & $3 \%$ & $0 \%$ \\
\hline \multicolumn{4}{|c|}{ TIEMPO DE RESIDENCIA } & & & \\
\hline & 5 A 15 años & 15 a 30 años & $<$ de 30 años & & & \\
\hline GUAYABAL & $25,20 \%$ & $21,50 \%$ & $53,30 \%$ & & & \\
\hline ROBLEDO & $38,60 \%$ & $32,50 \%$ & $28,90 \%$ & & & \\
\hline
\end{tabular}

Tabla 2. Resultados significativos de las espirometrías

\begin{tabular}{|l|l|l|l|}
\hline \multicolumn{4}{|c|}{ RESULTADOS } \\
\hline & GUAYABAL & ROBLEDO & RR \\
\hline ALTERACIÓN OBSTRUCTIVA & $56(14,7 \%)$ & $14(7 \%)$ & $2,252 \mathrm{P}<0,001,95 \%(1,220-4,158)$ mayor en Guayabal que en Robledo \\
\hline EPOC & $32(8,4 \%)$ & $7(3,6 \%)$ & $2,48995 \% \mathrm{p}<0,024(1,078-5,746)$ mayor en Guayabal que en Robledo \\
\hline
\end{tabular}

Tabla 3. Resultados significativos del cuestionario de síntomas

\begin{tabular}{|l|c|c|}
\hline \multicolumn{3}{|c|}{ RESULTADOS } \\
\hline & GUAYABAL & ROBLEDO \\
\hline BRONQUITIS CRÓNICA & $38(9,97 \%)$ & $8(4,06 \%)$ \\
\hline EXACERBACIONES & $31(8,1 \%)$ & $5(2,53 \%)$ \\
\hline
\end{tabular}


EPOC, BRONQUITIS CRÓNICA Y SÍNTOMAS RESPIRATORIOS, ASOCIADOS

a la CONTAMinaCión por PMio en la CiUdad de Medellín (Colombia)

\section{Discusión}

Estudios epidemiológicos han demostrado que las partículas en suspensión están asociadas a riesgo de mortalidad y hospitalización por EPOC.

Schikowskiet y colaboradores evaluaron la relación entre la exposición prolongada al aire contaminado por el tráfico y la función pulmonar y exacerbaciones de la EPOC. Los resultados mostraron que un aumento de $7 \mu \mathrm{g} / \mathrm{m}^{3}$ durante 5 años de PM10, se asoció con un odds ratio de 1,33 (95\% CI: 1.03-1.72) para la EPOC y que un aumento de $16 \mu \mathrm{g} / \mathrm{m}^{3}$ en promedio durante 5 años de dióxido de nitrógeno $\left(\mathrm{NO}_{2}\right)$ se asoció con un OR de 1.43 (IC 95\%: 1.23-1.66) para la EPOC. Además, las mujeres que viven a menos de 100 metros de una vía congestionada también tenían una función pulmonar disminuida significativamente; entre ellas, era de 1,79 veces más probabilidad de EPOC $(95 \%$ CI: 1.06-3.02) que para los que viven más lejos (23).

La contaminación atmosférica causa el $6 \%$ de la mortalidad total o más de 40.000 muertes al año. Más de 25.000 nuevos casos de bronquitis crónica (adultos); más de 290.000 casos de bronquitis (niños); y más de 16 millones días-persona de ausentismo de actividades (24). Los resultados del estudio MISA (meta análisis italiano del efecto a corto plazo de la contaminación atmosférica) mostraron un aumento de la mortalidad por todas las causas naturales asociados al aumento de la concentración de contaminantes de aire. Resultados similares se encontraron para mortalidad cardio-respiratoria, y admisiones hospitalarias por enfermedades respiratorias y cardíacas (25).

Baldacciet y colaboradores encontraron que la prevalencia de enfermedades, síntomas respiratorios, y los niveles de reactividad bronquial fueron más altos en el área urbana (26). Iversenet y colaboradores investigaron la epidemiología de las enfermedades respiratorias crónicas comunicadas en Escocia. Los resultados sugieren que quienes viven en un área rural tenían una menor prevalencia de asma, tos crónica, flema, disnea y sibilancias (significativamente) y otros trastornos respiratorios crónicos (no significativa). Alta frecuencia del tráfico de camiones en la calle de la residencia se asoció significativamente con mayores riesgos para la tos crónica o flema (OR 1,85, 95\% CI: 1.43-2.39) (27).

La edad de la población no es la única causa del incremento en la prevalencia del EPOC en los países industrializados en las últimas décadas. La exposición a agentes en los lugares de trabajo y la contaminación aérea juegan un papel en las exacerbaciones y en la patogénesis de la enfermedad respiratoria crónica, según un estudio alemán para EPOC, restringido a mujeres con la definición del criterio gold encontró que el EPOC era 1.79 veces más frecuente (95\% 1.06-3.02), para quienes vivían a menos de $100 \mathrm{mts}$ de la calle con un tráfico de 10.000 carros por día, que para quienes vivían más lejos de ésta. Ésto está de acuerdo con nuestros resultados donde se encontraron estos efectos en quienes viven a menos de $100 \mathrm{mts}$ de calles con 8640/día (6 carros/min) (28). En el estudio en Suecia, se encontró una asociación del tráfico automotor con la prevalencia de EPOC/ bronquitis crónica en adultos pero todavía se necesita investigar las razones de las inconsistencias vistas cuando los datos fueron estratificados por regiones $(29,30)$.

En el estudio PREPOCOL se concluyó que la prevalencia de EPOC en Medellín en la población $\geq 40$ años diagnosticada por espirometría es $13.5 \%$, este estudio incluyó los fumadores. La prevalencia de EPOC con base en el diagnóstico realizado por un médico es $5.8 \%$. La prevalencia de EPOC con base en los síntomas referidos por el paciente (bronquitis crónica) es $4.5 \%$. El número de casos diagnosticados por espirometría es mayor que el diagnóstico médico, el cual a su vez es mayor que el basado en los síntomas, lo cual sugiere un gran subregistro de enfermos en la población general de esta ciudad.

En Medellín realizamos un estudio en trabajadores en el centro de la ciudad, incluyendo también los fumadores, expuestos al tráfico automotor con los siguientes resultados: tos fue de $13 \%$ (55 personas), flema fue de $14.2 \%$ (57 personas), sibilancias "alguna vez" en la vida fue de $49.8 \%$ (188 personas), disnea fue de 38.5 $\%$ (154 personas), bronquitis crónica fue de 13.5\% (56 personas), asma fue de $13.5 \%$ (54 personas). El 52\% manifestaron haber fumado, en la actualidad fuman el $38.8 \%$ (155 personas) y el $26 \%$ manifiestan ser fumadores pasivos. El $41.5 \%$ eran vendedores ambulantes o de almacenes abiertos a la calle (31).

Esta investigación es una de las primeras en utilizar el criterio espirométrico estándar para definir el EPOC en relación con la contaminación aérea y sus resultados son en una población no fumadora. Al comparar estos estudios con nuestros resultados podemos observar que una proporción importante de EPOC podría deberse a la contaminación aérea por el tráfico automotor. 


\section{Conclusiones}

Los niveles de contaminación por PM10 están asociados con un aumento en la prevalencia de síntomas respiratorios de tos y flema, bronquitis crónica y EPOC en la ciudad de Medellín. Ésto indica que esta contaminación aérea no solo se asocia a efectos de corta duración sino también con efectos respiratorios de larga duración. Existe una asociación entre la prevalencia de EPOC y bronquitis crónica y los altos niveles de PM10 en Guayabal. Los niveles de PM10 en Guayabal, se encuentran por encima de los valores recomendados internacionalmente. Los niveles de PM10 se deben en un $90 \%$ al tráfico automotor. Los valores de PM10 en Guayabal se encuentran por debajo de la norma colombiana y por encima de la norma internacional de la OMS. Las emisiones de las fuentes móviles en la zona de Guayabal aportan el $37 \%$ del total emitido en el Valle de Aburrá.

\section{Recomendaciones}

Mejorar la calidad del diésel suministrado por parte de ECOPETROL para la ciudad de Medellín.

Integración del transporte público con el transporte masivo.

Realizar monitoreos permanentes de control tanto para las industrias como para los vehículos.

Realizar tamizajes para este tipo de patologías periódicamente.

Estructurar sistemas de alerta temprana de elevación de los niveles de PM10 para evitar el desarrollo y exacerbación de estas y otras patologías en la población.

Solicitar a las autoridades nacionales la revisión de la norma actual de calidad del aire.

\section{Agradecimientos}

El estudio fue financiado con recursos del presupuesto participativo de la secretaria de medioambiente de la alcaldía de la ciudad de Medellín y realizado con apoyo del grupo de investigación ambiental del CIDI y Facultad de Medicina de la Universidad Pontificia Bolivariana de Medellín.

\section{Referencias}

1. Halonen JI, Lanki T, Yli-Tuomi T, Kulmala M, Tiittanen P, Pekkanen J. Urban Air Pollution and Asthma and Copd Hospital Emergency Room Visits. Thorax. 2008; 63(7): 635-41.

2. Ling S, van Eeden S. Particulate matter air pollution exposure: role in the development and exacerbation of chronic obstructive pulmonary disease. J Chron Obstruct Pulmon Dis. 2009; 4: 233-243.

3. Kim JJ, Huen K, Adams S, Smorodinsky S, Hoats A, Malig B, et al., Residential traffic and children's respiratory health. Environ Health Perspect. 2008; 116(9): 1274-9.

4. Brauer M, Hoek G, van Vliet P, Meliefste K, Fischer PH, Wijga $A$, et al. Air pollution from traffic and the developmentof respiratory infections and asthmatic and allergic. Am J Respir Crit Care Med. 2002; 166(8): 1092-8.

5. Jerrett M, Shankardass K, Berhane K, Gauderman WJ, Künzli $\mathrm{N}$, Avol E, et al. Traffic-related air pollution and asthma onsetin children: a prospective cohort study with individual exposuremeasurement. Environ Health Perspect. 2008; 116(10): 1433-8.

6. Gauderman WJ, Vora H, McConnell R, Berhane K, Gilliland F, Thomas D, et al. Effect of exposure to traffic on lung development from 10 to 18 years of age: a cohort study. Lancet. 2007; 369(9561): 571-7.

7. Oftedal B, Brunekreef B, Nystad W, Madsen C, Walker SE, Nafstad P. Residential outdoor air pollution and lung function inschoolchildren. Epidemiology. 2008; 19(1): 129-37.

8. Morgenstern V, Zutavern A, Cyrys J, Brockow I, Gehring U, Koletzko S, et al. Respiratory health and individual estimated exposure to traffic-relatedair pollutants in a cohort of young children. Occup Environ Med. 2007; 64(1): 8-16.

9. Viegi G, Maio S, Pistelli F, Baldacci S, Carrozzi L. Epidemiology of chronic obstructive pulmonary disease: health effects of air pollution. Respirology. 2006; 11(5): 523-32.

10. Modig L, Järvholm B, Rönnmark E, Nyström L, Lundbäck B, Andersson, $\mathrm{C}$, et al. Vehicle exhaust exposure in an incident case-control study of adult asthma. EurRespir J. 2006; 28(1): 75-81.

11. Jacquemin B, Sunyer J, Forsberg B, Aguilera I, Briggs D, García-Esteban R, et al. Home Outdoor NO2 and New Onset of Self-Reported Asthma in Adults. Epidemiology. 2008; in press.

12. Wyler C, Braun-Fahrländer C, Künzli N, Schindler C, AckermannLiebrich U, Perruchoud AP, et al. Exposure to motor vehicle traffic and allergic sensitization. Epidemiology. 2000; 11(4): 450-6.

13. Zemp E, Elsasser S, Schindler C, Künzli N, Perruchoud AP, Domenighetti $G$, et al. Long-term ambientair pollution and respiratory symptoms in adults. Am J Respir Crit Care Med. 1999; 159(4 Pt 1): 1257-66.

14. Heinrich J, Topp R, Gehring U, Thefeld W. Traffic at residential address, respiratory health, and atopy in adults: the National German Health Survey 1998. Environ Res. 2005; 98(2): 240-9.

15. Schikowski T, Sugiri D, Ranft U, Gehring U, Heinrich J, Wichmann $\mathrm{HE}$, et al. Long-term air pollution exposure and living close to busy roads are associated with COPD in women. Respir Res. 2005, 6: 152

16. Sunyer J, Jarvis D, Gotschi T, Garcia-Esteban R, Jacquemin B, AguileraI, et al. Chronic bronchitis and urban air pollutionin an international study. Occup Environ Med. 2006; 63(12): 836-43.

17. Cesaroni G, Badaloni C, Porta D, Forastiere F, Perucci CA. Comparison between several indices of exposure to traffic-related air pollution and their respiratory health impact in adults. Occup Environ Med. 2008; 65(10): 683-90. 
18. Montnémery P, Bengtsson P, Elliot A, Lindholm LH, Nyberg $\mathrm{P}$, Löfdahl CG. Prevalence of obstructive lung diseases and respiratory symptoms in relation to living environment and socio-economic group. Respir Med. 2001; 95(9): 744-52.

19. Toro MV, Gomez JC, Orduz C. Informe preliminar a la Secretaria de Medio Ambiente. Medellín: Alcaldia de Medellín UPB; 2008.

20. Informes de RED AIRE. Medellín: RED AIRE; 1998-2007.

21. Orduz C. Asma Ocupacional. Medellín: Universidad Pontificia Bolivariana; 2000.

22. Celli BR, MacNee W. Standars for the diagnosis and treatment of pacients with COPD: A summary of the ATS/ERS position paper. EurResp J. 2004; 23: 932-46.

23. Schikowski T, Sugiri D, Ranft U, Gehring U, Heinrich J, Wichmann $\mathrm{HE}$, et al. Long-term air pollution exposure and living close to busy roads are associated with COPD in women. Respir Res. 2005; 6: 152-78. 34.

24. Anderson HR, Spix C, Medina S, Schouten JP, Castellsague J, Rossi G, et al. Air pollution and daily admissions for chronic obstructive pulmonary disease in 6 European cities: results from the APHEA project. Eur Respir J. 1997; 10: 1064-71.

25. Baldacci S, Carrozzi L, Viegi G, Giuntini C. Assessment of respiratory effect of air pollution: study design on general population samples. J Environ Pathol. Toxicol. Oncol. 1997; 16: 77-83.
26. Iversen L, Hannaford PC, Price DB, Godden DJ. Is living in a rural area good for your respiratory health? results from a cross-sectional study in Scotland. Chest. 2005; 128: 2059-67.

27. Ciccone G., Forastiere F., Agabiti N., Biggeri A., Bisanti L., Chellini E., et al. Road traffic and adverse respiratory effects in children.SIDRIA CollaborativeGroup. Occup. Environ. Med. 1998; 55: 771-8.

28. Lindgren A,Stroh E, Montnémery P, Nihlén U, Jakobsson K, Axmon A. Traffic-related air pollution associated with prevalence of asthma and COPD/chronic bronchitis. Int J Health Geogr. 2009; 8: 2.

29. Ling S, van Eeden S. Particulate matter air pollution exposure: role in the development and exacerbation of chronic obstructive pulmonary disease. J Chron Obstruct Pulmon Dis. 2009; 4: 233-243

30. Caballero A, Torres-Duque CA, Jaramillo C, Bolívar F, Sanabria F, Osorio P, et al. Prevalence of COPD in Five Colombian Cities Situated at Low, Medium, and High Altitude. Chest. 2008; 133(2); 343-349; published ahead of print October 20, 2007, doi:10.1378/chest.07-1361

31. Orduz C, Restrepo H. Informe al municipio de Medellín, prevalencia de síntomas respiratorios crónicos, bronquitis crónica y asma, en trabajadores expuestos a la contaminación del aire en el centro de la ciudad de Medellín. Medellín: Universidad de Antioquia; 2007. 